\title{
Electrocatalytic oxygen evolution reaction at a FeNi composite on a carbon nanofiber matrix in alkaline media
}

\author{
Xianghua An a, Dongyoon Shin a, Joey D. Ocon a, Jae Kwang Leeb, Young-il Son c, Jaeyoung Lee a,b,* \\ a Electrochemical Reaction and Technology Laboratory (ERTL), School of Environmental Science and Engineering, Gwangju Institute of Science and \\ Technology (GIST), Gwangju 500-712, South Korea \\ ${ }^{\mathrm{b}}$ Ertl Center for Electrochemistry and Catalysis, Research Institute for Solar and Sustainable Energies (RISE), Gwangju Institute of Science and Technology \\ (GIST), Gwangju 500-712, South Korea \\ c Future Environmental R\&D Office, Korea Environmental Industry \& Technology Institute (KEITI), Seoul 122-706, South Korea
}

\section{A R T I C L E I N F O}

Article history:

Received 11 April 2014

Accepted 28 April 2014

Published 20 June 2014

Keywords:

Oxygen evolution reaction

Carbon nanofiber

Iron

Nickel

Alkaline media

\begin{abstract}
A B S T R A C T
Non-noble metals such as $\mathrm{Fe}$ and Ni have comparable electrocatalytic activity and stability to that of Ir and $\mathrm{Ru}$ in an oxygen evolution reaction (OER). In this study, we synthesized carbon nanofibers with embedded FeNi composites (FeNi-CNFs) as OER electrocatalysts by a facile route comprising electrospinning and the pyrolysis of a mixture of metal precursors and a polymer solution. FeNi-CNFs demonstrated catalytic activity and stability that were better than that of $20 \mathrm{wt} \% \mathrm{Ir}$ on Vulcan carbon black in oxidizing water to produce oxygen in an alkaline media. Physicochemical and electrochemical characterization revealed that $\mathrm{Fe}$ and $\mathrm{Ni}$ had synergistic roles that enhanced OER activity by the uniform formation and widening of pores in the carbon structure, while the CNF matrix also contributed to the increased stability of the catalyst.
\end{abstract}

(C) 2014, Dalian Institute of Chemical Physics, Chinese Academy of Sciences. Published by Elsevier B.V. All rights reserved.

\section{Introduction}

Oxygen electrocatalysis has attracted much attention because the reactions involving oxygen play an important role in almost all electrochemical technologies and processes [1,2], which include water electrolyzers, regenerative fuel cells, and rechargeable metal-air batteries where the oxygen evolution reaction (OER) is the rate determining step due to its slow kinetics. Bulk metal oxides such as $\mathrm{RuO}_{2}, \mathrm{IrO}_{2}, \mathrm{NiO}$, and perovskites are active catalysts for the OER. $\mathrm{RuO}_{2}$ and $\mathrm{IrO}_{2}$ exhibited the lowest overpotential among these and are the best catalysts for the OER. However, Ru and Ir are among the most expensive materials, and their oxides lack stability due to their poor resistance to corrosion in the highly oxidative environment of oxygen evolution. In addition to cost, the poor stability of the metal oxides at high anodic potential makes it difficult to use them in industrial applications. Therefore, finding cost-effective OER electrocatalysts with comparable activities to $\mathrm{Ru}$ and Ir oxides but with better stability is the key to the challenge of commercial application.

Significant efforts have been devoted to develop catalysts to replace the noble metal oxide catalysts, preferably with cheaper non-noble metals. Transition metals such as $\mathrm{Fe}, \mathrm{Co}$, and $\mathrm{Ni}$ (elements of the iron triad) with amorphous or spinel oxide structures have been investigated as alternative catalysts because they are chemically stable in alkaline media and they showed comparable OER activity to the Ru- and Ir-based catalysts [3-9]. In particular, alloys of $\mathrm{Ni}$ and Fe showed excellent performance as highly active and corrosion-resistant catalysts, and are good choices for inexpensive OER catalysts in alkaline

* Corresponding author. Tel: +82-62-7152440; Fax: +82-62-7152434; E-mail: jaeyoung@gist.ac.kr 
media [9-12]. Further studies on improving the activity and stability of the iron triad-based catalysts by using them with carbon materials, such as carbon nanotubes (CNTs) and graphene, have been made $[7,9,13,14]$. The combination with carbon materials has resulted in increased activity and enhanced stability towards OER, which was primarily due to the reduced corrosion kinetics of the metal particles.

In this paper, we used carbon nanofibers (CNFs) easily fabricated by the electrospinning and pyrolysis of the precursor mixture as a carbon matrix, in which $\mathrm{Fe}$ and $\mathrm{Ni}$ particles are embedded. From our previous experience, we expected the CNFs to provide a suitable reacting medium for the stable formation of individual metal and metallic alloy particles in a highly dispersed state and that the embedded particles would be protected by the carbon layer to enhance their stability [15-17]. We tested the activity and stability of FeNi-CNFs in the OER, compared these with an Ir-based electrocatalyst, and elucidated the reason for the improved electrochemical performance using physical and chemical characterization.

\section{Experimental}

\subsection{Catalyst preparation}

The metal-containing CNFs (Me-CNFs) were synthesized by electrospinning a mixture of metal precursors and a polymer solution to form a polymeric nanofiber web. To prepare the polymer solution, $4 \mathrm{~g}$ of polyacrylonitrile (PAN, Sigma-Aldrich) was dissolved in $36 \mathrm{~g}$ of $\mathrm{N}, \mathrm{N}$-dimethylformamide (DMF, Junsei) at $60-80{ }^{\circ} \mathrm{C}$ for several hours. Then, the polymer solution was mixed with the metal precursors, $\mathrm{Ni}(\mathrm{II})$ acetylacetonate (Ni(acac)2, Sigma-Aldrich) and Fe(III) acetylacetonate (Fe(acac) $)_{3}$, Sigma-Aldrich). This method favored the formation of a carbon protection layer during carbonization, which encapsulated the metal structure because the central metal is surrounded by carbon rings in the metal precursor above $[18,19]$. The total amount of metal precursor in the polymer solution was $1.2 \mathrm{~g}$, and the weight ratios of the $\mathrm{Ni}$ and Fe precursors were 1:0 for Ni-CNFs, 0:1 for Fe-CNFs, 9:1 for FeNi(1:9)-CNFs, and 5:5 for FeNi(5:5)-CNFs. The FeNi composite-containing CNFs were named according to the metal precursor ratio. Me-PAN mixtures were fed at a flow rate of $5 \mathrm{~mL}$ $\mathrm{h}^{-1}$ for $6 \mathrm{~h}$ by a syringe pump with a multi-nozzle. Then, a high electric voltage of $24 \mathrm{kV}$ was applied between the syringe and collector in the electrospinning process. All processes were performed at room temperature $\left(25^{\circ} \mathrm{C}\right)$ and below $30 \%$ relative humidity (RH). The spun PAN nanofibers were simultaneously pyrolyzed in air and $\mathrm{N}_{2}$ environments as the two-step stabilization stage and carbonization stage, respectively [15-17].

\subsection{Characterization}

The electrocatalysts were characterized by X-ray diffraction (XRD, Rigaku Miniflex II) to determine their bulk structure. The morphology of the electrocatalysts was examined with scanning transmission/transmission electron microscopy (STEM/
TEM; Philips TECNAI F20, equipped with energy dispersive X-ray spectroscopy (EDS)) in the Gwangju Center of the Korea Basic Science Institute (KBSI), Republic of Korea. The specific surface area and pore size distribution of the Me-CNFs were calculated from $\mathrm{N}_{2}$ adsorption isotherms using the BET and $\mathrm{BJH}$ methods, respectively (Belsorp-max, Japan).

\subsection{Activity test}

The electrochemical properties and OER activity of the Ni-CNFs, Fe-CNFs, FeNi(1:9)-CNFs, and FeNi(5:5)-CNFs catalysts were measured using cyclic voltammetry (CV) and chronoamperometry (CA) in a three electrode cell connected to a potentiostat/galvanostat (Biologic, VSP). We used a smooth $\mathrm{Pt}$ wire as the counter electrode and $\mathrm{Hg} / \mathrm{HgO}$ as the reference electrode. The catalyst ink was prepared using $10 \mathrm{mg}$ of catalyst dispersed in $10 \mu \mathrm{L} 10 \mathrm{wt} \%$ Nafion solution (Sigma-Aldrich), $4 \mathrm{~mL}$ DI water and $1 \mathrm{~mL}$ isopropyl alcohol. After ultrasonication for at least $30 \mathrm{~min}$, a $25 \mu \mathrm{L}$ aliquot of the suspension was dropped onto a glassy carbon (GC) disk electrode $(0.2475$ $\mathrm{cm}^{2}$ ) using a micropipette, which gave a catalyst loading of 200 $\mu \mathrm{g} \mathrm{cm}-2$. For OER activity and stability comparison, a $20 \mathrm{wt} \%$ Ir on Vulcan carbon black catalyst (Premetek Co.) was used for comparing with the activities of the Me-CNFs.

The OER activity of the Me-CNFs was measured by sweeping the potential from 0 to $1.2 \mathrm{~V}$ versus $\mathrm{Hg} / \mathrm{HgO}$ at a scan rate of $100 \mathrm{mV} \mathrm{s}^{-1}$ and a rotating rate of $1600 \mathrm{rpm}$ in $0.1 \mathrm{~mol} \mathrm{~L}^{-1} \mathrm{KOH}$ (Sigma-Aldrich) electrolyte. The electrolyte was saturated with ultrapure $\mathrm{N}_{2}$ at a flow rate of $20 \mathrm{~mL} \mathrm{~min}^{-1}$ at room temperature. CA was performed at $0.9 \mathrm{~V}$ vs $\mathrm{Hg} / \mathrm{HgO}$ electrode for $90 \mathrm{~min}$ to observe the long term stability of the catalysts.

\section{Results and discussion}

The XRD patterns of the Me-CNFs confirmed that changes in the bulk crystalline structure depended on the ratio of $\mathrm{Fe}$ and Ni. As shown in Fig. 1, in the Ni-containing CNFs, i.e. Ni-CNFs, FeNi(1:9)-CNFs, and FeNi(5:5)-CNFs, there were three peaks at $44.5^{\circ}, 51.8^{\circ}$, and $76.4^{\circ}$, which were assigned to the (111), (200), and (220) diffraction planes of metallic $\mathrm{Ni}$, respectively. In contrast, iron carbide $\left(\mathrm{Fe}_{3} \mathrm{C}\right)$, not metallic $\mathrm{Fe}$, was the dominant phase in the Fe-containing CNFs. All the samples exhibited a peak at $26^{\circ}$, which was attributed to the (002) plane of graphitic carbon derived from the turbostratic carbon structure of CNFs. However, the degree of graphitization of the carbon in the Me-CNFs varied depending on the type of metal. This phenomenon of catalytic graphitization usually occurs when carbon is heat-treated in the presence of certain metals (e.g., Al, Co, $\mathrm{Fe}$, and $\mathrm{Ni}$ ) or inorganic compounds (e.g., phthalimide and pyromellitic dianhydride) [20]. Of the inorganic and organic compounds for catalytic graphitization, Fe commonly combines with carbon as a carbide in forming graphitic carbon, while $\mathrm{Ni}$ melts and dissolves in the amorphous carbon in the process, which is termed the dissolution-precipitation mechanism. Zhou et al. [21] reported that the catalytic effect of a Ni-Fe alloy on the graphitization of carbon fibers was better than that of $\mathrm{Ni}$ or $\mathrm{Fe}$ at the same temperature. The diffractograms of the Me-CNFs 


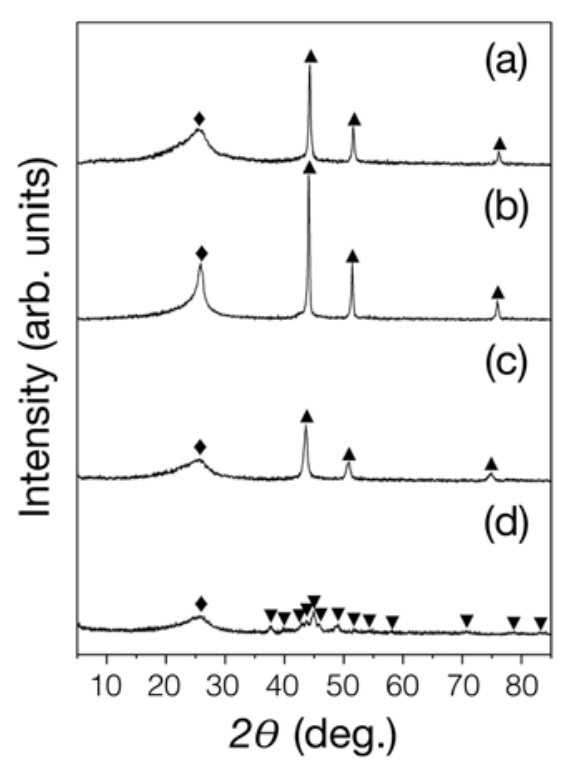

Fig. 1. X-ray diffractograms of (a) Ni-CNFs, (b) FeNi(1:9)-CNFs, (c) FeNi(5:5)-CNFs, and (d) Fe-CNFs. The peaks are marked by the following symbols: Graphite, $\Delta \mathrm{Ni}$, and $\boldsymbol{\nabla} \mathrm{Fe}_{3} \mathrm{C}$.

suggested that the Fe atoms were well bonded with the carbon structure, allowing the dispersion of $\mathrm{Fe}$ atoms in the carbon matrix to form $\mathrm{Fe}_{3} \mathrm{C}$. The formation of $\mathrm{Fe}_{3} \mathrm{C}$ would lead to smaller pores, which will be demonstrated later in the PSD results. On the other hand, with $\mathrm{Ni}$ and the FeNi alloy, wider pores in the carbon were formed than was the case with the elements that form the carbides, such as $\mathrm{Fe}, \mathrm{Al}$, and $\mathrm{Si}$. This was because they followed the dissolution-precipitation mechanism during catalytic graphitization $[20,22]$.

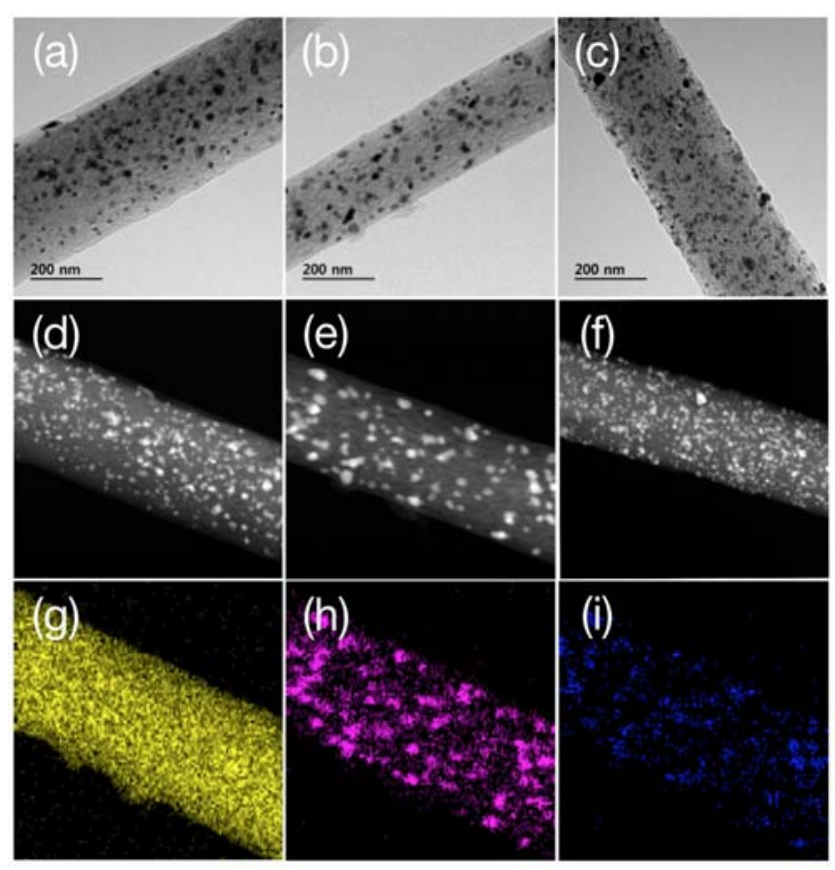

Fig. 2. TEM images of (a) Ni-CNFs, (b) FeNi(1:9)-CNFs, and (c) Fe-CNFs. STEM images of (d) Ni-CNFs, (e) FeNi(1:9)-CNFs, and (f) Fe-CNFs. EDS element mapping for (g) carbon, (h) nickel, and (i) iron in the FeNi(1:9)-CNFs electrocatalyst.
To confirm the morphology of the synthesized Me-CNFs, we performed STEM/TEM analyses. The nanofiber diameters were in the range of 100-500 $\mathrm{nm}$, which was similar to the CNF sizes we observed in our previous studies [15-17]. As displayed in Fig. 2(a-c), most of the metal nanoparticles (NPs) were embedded homogeneously in the CNF matrix. As exhibited in the TEM images, the $\mathrm{Fe}_{3} \mathrm{C}$ particles were well dispersed as compared to the $\mathrm{Ni}$ and FeNi particles, which was expected from the XRD results. In the EDS mapping for FeNi(1:9)-CNFs, the Ni NPs were well distributed together with the Fe NPs in the CNFs (Fig. 2(g-i)). This could indicate the formation of a FeNi alloy, complementing the XRD results. As seen in the STEM images (Fig. 2(d-f)), the pore distribution of the Me-CNFs varied a lot depending on the type of metal embedded in it. Ni-containing CNFs, i.e., Ni-CNFs and FeNi(1:9)-CNFs, showed clearly the presence of pores in the carbon matrix. The pore size of FeNi(1:9)-CNFs was slightly larger than that of Ni-CNFs, as shown in Fig. 2(d-e). In contrast, Fe-CNFs did not seem to have any visible pores (Fig. 2(f)).

The $\mathrm{N}_{2}$ adsorption isotherms of the Ni-CNFs, FeNi(1:9)CNFs, FeNi(5:5)-CNFs, and Fe-CNFs electrocatalysts gave BET surface areas of 207.57, 256.63, 377.05, and $367.97 \mathrm{~m}^{2} \mathrm{~g}^{-1}$, respectively (Fig. 3(a)). The increase in the specific surface area with the amount of Fe can be explained by the enhanced pore formation around the metal particles. This was consistent also with the highly dispersed nature of the $\mathrm{Fe}_{3} \mathrm{C}$ NPs in the Fe-containing CNFs in above discussion. The pore size distribution analysis based on the desorption branch (Fig. 3(b)) showed that the Fe-CNFs has a narrow pore size distribution of 2-3 nm. On the other hand, the Ni-containing samples, i.e., Ni-CNFs, FeNi(1:9)-CNFs, and FeNi(5:5)-CNFs, exhibited a wider spread of pore sizes. In particular, the FeNi(1:9)-CNFs sam-
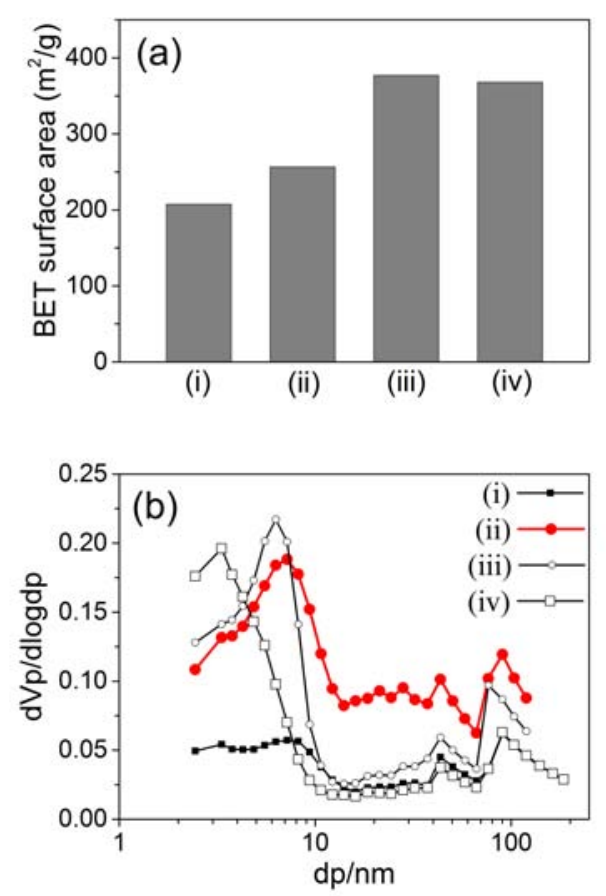

Fig. 3. (a) BET surface area and (b) BJH plots for (i) Ni-CNFs, (ii) FeNi(1:9)CNFs, (iii) FeNi(5:5)-CNFs, and (iv) Fe-CNFs. 
ple had a peak pore size at $9 \mathrm{~nm}$ and evenly distributed pores of all sizes. It was evident from these results that Fe played a crucial role in forming pores uniformly, while Ni gave bigger pores in the CNF matrix. We believe that the increased number of large mesopores in FeNi-CNFs effectively enhanced the OER activity due to increased accessible active sites.

Figure 4 shows the OER performance of the Me-CNFs and a comparison with $20 \mathrm{wt} \% \mathrm{Ir} / \mathrm{C}$ as the reference catalyst. The activity and stability were measured using repeated voltammetric cycling in a $\mathrm{N}_{2}$-purged $0.1 \mathrm{~mol} \mathrm{~L}^{-1} \mathrm{KOH}$ aqueous electrolyte with a GC rotating disk electrode at a rotating rate of 1600 rpm to instantly remove generated oxygen bubbles. As shown in Fig. 4(a-b), the monometallic CNFs samples, i.e., Ni-CNFs and Fe-CNFs, showed low activity and extremely poor stability. The Ni-CNFs sample demonstrated low activity initially but gave a much higher activity after 100 cycles, which possibly resulted from increased electrolyte access to the $\mathrm{Ni}$ active sites. In contrast, the Fe-CNFs sample gave high initial activity but suffered from severe corrosion after 100 cycles. On the other hand, the FeNi(1:9)-CNFs sample exhibited a comparable OER current density to that of $20 \mathrm{wt} \% \mathrm{Ir} / \mathrm{C}$ at $1.2 \mathrm{~V}$ vs $\mathrm{Hg} / \mathrm{HgO}$, and it sustained its activity well over 100 cycles (Fig. 4(c,e)). Furthermore, its OER activity was well maintained during every scan, as exhibited in Fig. 4(f), even when we increased the applied potential up to $1.5 \mathrm{~V}$ vs $\mathrm{Hg} / \mathrm{HgO}$ in order to see the stability of the FeNi(1:9)-CNFs sample under a harsher environmental condition. The FeNi(5:5)-CNFs sample showed a similar tendency to the FeNi(1:9)-CNFs sample, but it gave a slightly lower

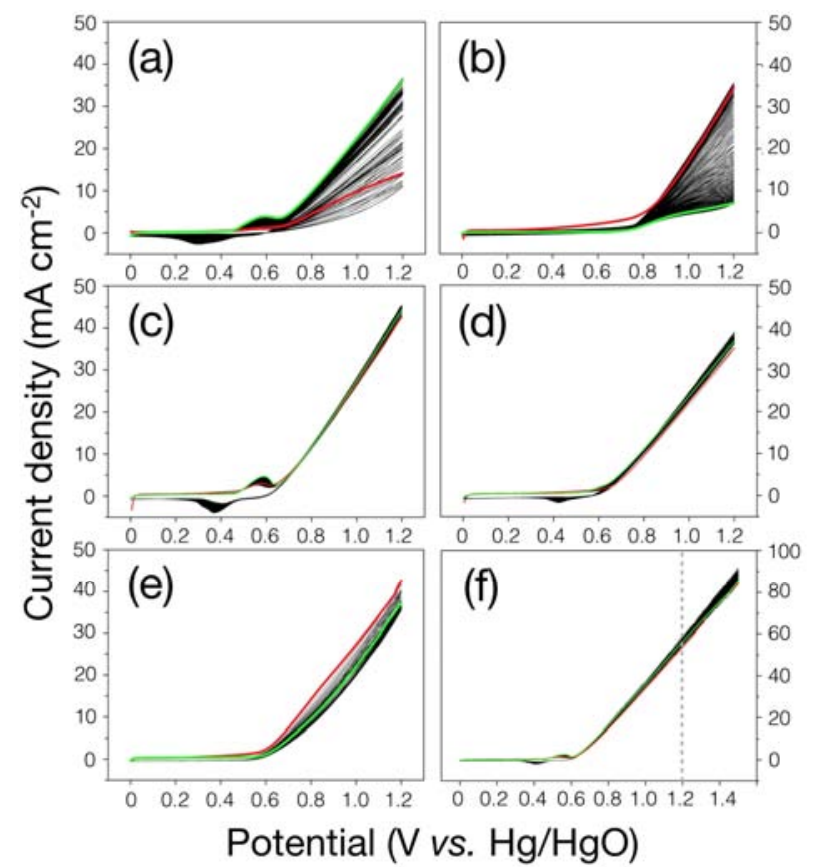

Fig. 4. Cyclic voltammograms of (a) Ni-CNFs, (b) Fe-CNFs, (c) FeNi(1:9)-CNFs, (d) FeNi(5:5)-CNFs, and (e) 20 wt\% Ir/C between 0.0 and $1.2 \mathrm{~V}$. (f) FeNi(1:9)-CNFs was subjected to a further increased anodic potential of $1.5 \mathrm{~V}$ to see the stability under a harsher condition. Dashed line indicates the limited potential of $1.2 \mathrm{~V}$. N2-purged $0.1 \mathrm{~mol}$ $\mathrm{L}^{-1} \mathrm{KOH}$ aqueous solution with GC RDE at a rotating rate of $1600 \mathrm{rpm}$ and a scan rate of $100 \mathrm{mV} \mathrm{s}^{-1}$. Number of scan: 100 cycles. Red and green lines show the 1 st and 100th scan of each catalyst.

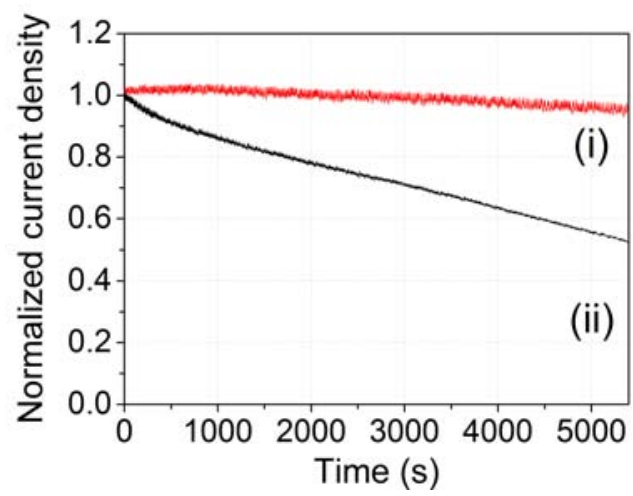

Fig. 5. Chronoamperometry curves of (i) FeNi(1:9)-CNFs and (ii) 20 wt $\% \mathrm{Ir} / \mathrm{C}$ in a $\mathrm{N}_{2}$-purged $0.1 \mathrm{~mol} \mathrm{~L}^{-1} \mathrm{KOH}$ aqueous solution with GC RDE at a rotating rate of $1600 \mathrm{rpm}$ and a scan rate of $100 \mathrm{mV} \mathrm{s}^{-1}$.

OER activity and stability than FeNi(1:9)-CNFs sample (Fig. 4(d)). The prolonged high activity of the FeNi-CNFs samples, in contrast to the increasing and decreasing activities of the Ni-CNFs and Fe-CNFs samples, further indicated that a critical number of pores were readily available due to the uniform dispersion of pore size, so that the electrolyte can readily access the OER active sites (see Fig. 3(b)). This higher activity of the FeNi(1:9)-CNFs revealed that Fe and Ni exhibited a synergistic role in enhancing the OER activity, which was through the uniform formation and widening of the pores in the carbon structure. The improvement in the OER activity due to the combination of $\mathrm{Ni}$ and $\mathrm{Fe}$ was in agreement with a previous study by Gong et al. [9] where a FeNi-layered double hydroxide/carbon nanotube (FeNi-LDH/CNT) catalyst exhibited better electrochemical kinetics than $20 \mathrm{wt} \% \mathrm{Ir} / \mathrm{C}$.

To confirm the stability of the FeNi(1:9)-CNFs electrocatalyst in a reactive oxygen evolution environment, we performed $\mathrm{CA}$ at $0.9 \mathrm{~V}$ vs $\mathrm{Hg} / \mathrm{HgO}$ for the FeNi(1:9)-CNFs and $20 \mathrm{wt} \% \mathrm{Ir} / \mathrm{C}$ catalysts (Fig. 5). After an electrolysis time of $90 \mathrm{~min}$, the current density of FeNi(1:9)-CNFs had only decreased by $5 \%$, in contrast to the $48 \%$ reduction with $20 \mathrm{wt} \% \mathrm{Ir} / \mathrm{C}$. This was good evidence that the FeNi(1:9)-CNFs catalyst had an extremely stable OER activity, as shown in the slower activity decline than $20 \mathrm{wt} \% \mathrm{Ir} / \mathrm{C}$. Since Ni is one of the most stable OER-active materials in alkaline media, we can assume that this was due to $\mathrm{Ni}$ being the main composite material in the FeNi(1:9)-CNFs catalyst. In addition, the CNF matrix, which envelopes the metal particles, would also have prevented the deactivation of the metal active sites.

\section{Conclusions}

Metal-containing CNF electrocatalysts were prepared by a facile synthesis comprising electrospinning and pyrolysis of a polymer-metal precursor mixture. The FeNi(1:9)-CNFs catalyst showed better OER activity in alkaline water oxidation than the monometallic CNFs and a commercial 20 wt $\%$ Ir/C catalyst. Fe and $\mathrm{Ni}$ exhibited synergistic effect for the better activity in OER, with Fe having the role of forming the pores and $\mathrm{Ni}$ as responsible for the optimum distribution of pore sizes to facilitate the access of reactants to the OER active sites. These studies can 


\title{
Graphical Abstract
}

\author{
Chin. J. Catal., 2014, 35: 891-895 doi: 10.1016/S1872-2067(14)60127-3
}

\section{Electrocatalytic oxygen evolution reaction at a FeNi composite on a carbon nanofiber matrix in alkaline media}

Xianghua An, Dongyoon Shin, Joey D. Ocon, Jae Kwang Lee, Young-il Son, Jaeyoung Lee*

Gwangju Institute of Science and Technology (GIST), South Korea;

Korea Environmental Industry \& Technology Institute (KEITI), South Korea

Synergism between $\mathrm{Fe}$ and $\mathrm{Ni}$ embedded in carbon nanofibers resulted in an electrocatalyst with enhanced electrocatalytic activity and stability for oxygen evolution from water.

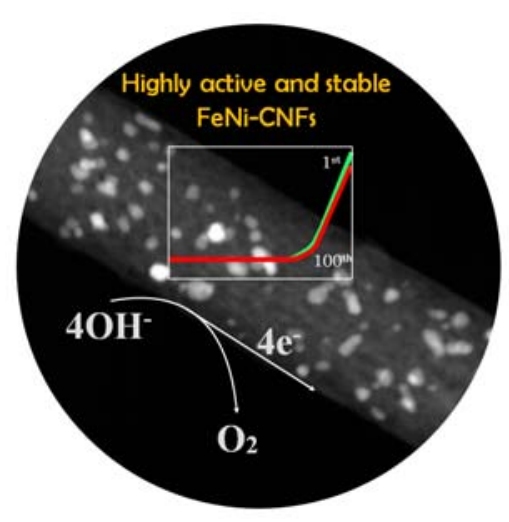

lead to better and inexpensive oxygen evolution electrocatalysts with superior activity and stability to noble metal-based catalysts for energy efficient chemical energy storage and conversion cells in alkaline media.

\section{Acknowledgments}

J. Lee is deeply indebted to the Alexander von Humboldt Foundation fellowship for experienced researchers (1141065). This work was also supported by the Core Technology Development Program of the Research Institute for Solar and Sustainable Energies (RISE), Gwangju Institute of Science and Technology.

\section{References}

[1] Kinoshita K. Electrochemical Oxygen Technology. Berkeley: Wiley, 1992

[2] Lee J, Jeong B, Ocon J D. Curr Appl Phys, 2013, 13: 309

[3] Osaka T, Ishibashi H, Endo T, Yoshida T. Electrochim Acta, 1981, 26: 339

[4] Lyons M E G, Brandon M P. Int J Electrochem Sci, 2008, 3: 1386

[5] Doyle R L, Godwin I J, Brandon M P, Lyons M E G. Phys Chem Chem Phys, 2013, 15: 13737

[6] Bockris J O, Otagawa T. J Electrochem Soc, 1984, 131: 290

[7] Liang Y Y, Li Y G, Wang H L, Zhou J G, Wang J, Regier T, Dai H J. Nat
Mater, 2011, 10: 780

[8] Kim J W, Lee J K, Phihusut D, Yi Y M, Lee H J, Lee J.J Phys Chem C, 2013, 117: 23712

[9] Gong M, Li Y G, Wang H L, Liang Y Y, Wu J Z, Zhou J G, Wang J, Regier T, Wei F, Dai H J.J Am Chem Soc, 2013, 135: 8452

[10] Kleiman-Shwarsctein A, Hu Y S, Stucky G D, McFarland E W. Electrochem Commun, 2009, 11: 1150

[11] Kim K H, Zheng J Y, Shin W, Kang Y S. RSC Adv, 2012, 2: 4759

[12] Lu Z Y, Xu W W, Zhu W, Yang Q, Lei X D, Liu J F, Li Y P, Sun X M, Duan X. Chem Commun, 2014, in press, doi: 10.1039/C4CC01625D

[13] Mette K, Bergmann A, Tessonnier J P, Hävecker M, Ressler T, Schlögl R, Strasser P, Behrens M. ChemCatChem, 2012, 4: 851

[14] Wang J, Zhong H X, Qin Y L, Zhang X B. Angew Chem Int Ed, 2013, 52: 5248

[15] Shin D, Jeong B, Mun B S, Jeon H, Shin H J, Baik J, Lee J.J Phys Chem C, 2013, 117: 11619

[16] Jeong B, Shin D, Jeon H, Ocon J D, Mun B S, Baik J, Shin H J, Lee J. ChemSusChem, 2014, in press, doi: 10.1002/cssc.201301374

[17] Uhm S, Jeong B, Lee J. Electrochim Acta, 2011, 56: 9186

[18] Barakat N A M, Kim B, Park S J, Jo Y, Jung M H, Kim H Y. J Mater Chem, 2009, 19: 7371

[19] Deng D H, Yu L, Chen X Q, Wang G X, Jin L, Pan X L, Deng J, Sun G Q, Bao X H. Angew Chem Int Ed, 2013, 52: 371

[20] Kinoshita K. Carbon: Electrochemical and Physicochemical Properties. Berkeley: Wiley, 1988

[21] Zhou H H, Peng Q L, Huang Z H, Yu Q, Chen J H, Kuang Y F. Trans Nonferrous Met Soc China, 2011, 21: 581

[22] Käärik M, Arulepp M, Karelson M, Leis J. Carbon, 2008, 46: 1579 